\title{
Impacto da Política Nacional de Saúde Bucal - Programa Brasil Sorridente - sobre a provisão de serviços odontológicos no Estado do Rio de Janeiro
}

\author{
Impact of the Brazilian Oral Health Policy - Programa \\ Brasil Sorridente - on the provision of public dental services in the \\ State of Rio de Janeiro
}

\author{
Angela Scarparo ${ }^{1}$, Thabata Cristy Zermiani ${ }^{2}$, Rafael Gomes Ditterich ${ }^{3}$, \\ Márcia Helena Baldani Pinto ${ }^{4}$
}

\begin{abstract}
Resumo
Esse estudo tem por objetivo avaliar o impacto da atual Política Nacional de Saúde Bucal, conhecida como Programa Brasil Sorridente (PBS), sobre a provisão de serviços odontológicos no Estado do Rio de Janeiro. Para tanto, foi investigada a evolução na disponibilidade de serviços focalizados pela PBS, entre o ano de implantação e 2010. Os itens analisados foram: Atenção Básica - Estratégia Saúde da Família: evolução no número de equipes de saúde bucal, e procedimentos (ações básicas, primeira consulta programática, preventivos coletivos, preventivos individuais, cirúrgicos) por habitante; Atenção Especializada: identificação dos municípios que possuem Centros de Especialidades Odontológicas - CEO, verificação da evolução dos procedimentos endodônticos e periodontais; e Fluoretação da águas: presença ou não de água fluoretada. De acordo com os resultados observou-se evolução nos indicadores analisados, os quais foram distintos segundo o porte demográfico dos municípios. Conclui-se que a PNSB favoreceu a organização dos serviços de saúde bucal nos municípios fluminenses, embora ainda existam desafios a serem superados.

Palavras-chave: saúde bucal; avaliação em saúde; política de saúde; atenção primária à saúde; atenção secundária à saúde.
\end{abstract}

\begin{abstract}
This study aims to assess the impact of the current Brazilian Oral Health Policy (BOHP), named Programa Brasil Sorridente (PBS) on the provision of public dental services in the State of Rio de Janeiro. Therefore, the evolution in the availability of services focused on PBS, between 2004 and 2010, was investigated. The items discussed were: Primary Care - Family Health Strategy: evolution in the number of oral health teams, and procedures (basic actions, the first programmatic consultation, preventive collective, individual preventive, surgical), per capita; Specialized Care: identification of municipalities that have specialized dental clinics - CEO, checking the progress of periodontal and endodontic procedures, and Water fluoridation: presence or absence of fluoridated water. According to the results it was observed changes in the indicators analyzed, which were different according to the municipalities demographic size. It is concluded that the BOHP favored the organization of oral health services in the cities of Rio de Janeiro, although there are still challenges to overcome.
\end{abstract}

Keywords: oral health; health evaluation; health policy; primary health care; secondary care.

\footnotetext{
${ }^{1}$ Faculdade de Odontologia, Polo Universitário de Nova Friburgo, Universidade Federal Fluminense (UFF) - Nova Friburgo (RJ), Brasil.

${ }^{2}$ Programa de Pós-graduação em Políticas Públicas, Universidade Federal do Paraná (UFPR) - Curitiba (PR), Brasil.

${ }^{3}$ Departamento de Odontologia, Universidade Estadual de Ponta Grossa (UEPG) - Ponta Grossa (PR), Brasil.

${ }^{4}$ Departamento de Saúde Comunitária, Universidade Federal do Paraná (UFPR) - Curitiba (PR), Brasil.

Trabalho realizado na Universidade Federal Fluminense (UFF) - Nova Friburgo (RJ), Brasil.

Endereço para correspondência: Angela Scarparo - Universidade Federal Fluminense, Rua Dr Silvio Henrique Braune - 22 - Centro - CEP: 28625-250 -

Nova Friburgo (RJ), Brasil - Email: angelascarparo@id.uff.br

Fonte de financiamento: nenhuma.

Conflito de interesses: nada a declarar.
} 


\section{INTRODUÇÃO}

Durante anos a Odontologia esteve à margem das políticas públicas de saúde. O modelo de assistência odontológica estatal era centrado no atendimento a grupos prioritários (crianças em idade escolar) e urgências, e gerava um quadro de intensa exclusão do acesso e baixo impacto sobre os índices epidemiológicos de doenças bucais ${ }^{1}$. Com a criação do Sistema Único de Saúde (SUS), em 1988, buscou-se romper com esse modelo vigente, no entanto, somente algumas experiências isoladas conseguiram ampliar o acesso, desenvolver ações de promoção e ofertar serviços mais complexos ${ }^{2}$.

De acordo com o sanitarista Gilberto Pucca Jr. ${ }^{3}$, Coordenador Nacional de Saúde Bucal, a assistência odontológica pública brasileira estava sendo prioritariamente desenvolvida para crianças e incluía procedimentos simples, tais como: exodontias, dentística restauradora, pequenas cirurgias e aplicações de flúor; sendo que apenas 3,3\% dos procedimentos realizados eram considerados especializados. Em contrapartida, constatou-se que, na população adulta, o quadro geral era lastimável, isto é, menos de $22 \%$ da população adulta apresentava boas condições gengivais, sendo que esta porcentagem, em idosos, diminuía para 10\%. Além disso, 30 milhões de brasileiros estavam edêntulos e 28 milhões nunca tinham ido ao dentista ${ }^{3,4}$.

Diante destes resultados, o Governo Federal, em 2004, apresentou uma nova Política Nacional de Saúde Bucal (PNSB), o Programa Brasil Sorridente (PBS). As bases deste Programa foram discutidas em uma reunião realizada em São Paulo em 2002, resultando no documento intitulado "Fome Zero e boca cheia de dentes", o qual continha as principais decisões da reunião, um diagnóstico da situação de saúde bucal no país, bem como as ações de saúde bucal que deveriam ser realizadas no âmbito do governo que se iniciava ${ }^{5}$.

O Programa Brasil Sorridente é constituído por “[...] medidas que têm como objetivo garantir as ações de promoção, prevenção e recuperação da saúde bucal dos brasileiros, entendendo que esta é fundamental para a saúde e qualidade de vida da população"'. Apresenta como principal objetivo, a reorganização da prática, bem como a qualificação das ações e serviços ofertados, por meio de uma série de ações em saúde bucal direcionadas aos cidadãos de todas as idades, com ampliação do acesso ao tratamento odontológico aos brasileiros, por meio do Sistema Único de Saúde (SUS) 6 .

As diretrizes da PNSB surgem, então, com o propósito de oferecer a viabilização de fluoretação das águas de abastecimento público, a reorganização da atenção básica por meio da Estratégia Saúde da Família (ESF) e da atenção odontológica especializada através da implantação de Centros de Especialidades Odontológicas (CEOs) e dos Laboratórios Regionais de Próteses Dentárias (LRPD) ${ }^{4,7}$.
Sendo assim, considerando-se a realidade de saúde bucal da população brasileira na época e as metas estabelecidas pelo PNSB evidencia-se a necessidade de uma análise mais aprofundada sobre o impacto deste programa sobre a provisão de serviços odontológicos, 6 anos após sua implantação em 2004. Dessa forma, este estudo teve por objetivo avaliar o impacto da atual Política Nacional de Saúde Bucal, conhecida como Brasil Sorridente (PBS), sobre a provisão de serviços odontológicos no Estado do Rio de Janeiro, no período compreendido entre 2004 e 2010.

\section{METODOLOGIA}

Este estudo é classificado como ecológico ou de correlação, de natureza quantitativa, e envolveu todos os 92 municípios do Estado do Rio de Janeiro. Para avaliar a evolução da implantação do PBS no Estado, os itens analisados contemplaram as principais linhas de atuação da política, quais sejam: a Atenção Básica e a evolução da Estratégia Saúde da Família; a Atenção especializada e a implantação dos Centros de Especialidades Odontológicas (CEOs); e a evolução da fluoretação das águas de abastecimento público.

Foi realizada uma busca investigativa nas seguintes bases de dados: Instituto Brasileiro de Geografia e Estatística (IBGE), Ministério da Saúde/Departamento de Atenção Básica (MS/DAB), Fundação Nacional de Saúde (FUNASA), Sala de Situação em Saúde do Ministério da Saúde, e o Banco de Dados do Sistema de Informação Ambulatorial do Sistema Único de Saúde (SIA/SUS).

Em se tratando do item Atenção Básica e Estratégia Saúde da Família, foram obtidos os indicadores de evolução no número de equipes de saúde bucal, no período compreendido entre 2004 e 2010, e os procedimentos (ações básicas, primeira consulta programática, preventivos coletivos, preventivos individuais, cirúrgicos), por habitante, para os anos de 2004 (início do PBS) e 2007. Cumpre ressaltar que o ano de 2010 não foi incluído devido à alteração na base de dados do SIA-SUS de 2007 para 2008 e falta de correspondência com alguns dos procedimentos.

Para coleta dos dados referentes a este item, foram utilizados os dados do IBGE e do Sistema de Informação Ambulatorial (SIA-SUS), no qual foram selecionadas as categorias de "ações básicas em odontologia" e "1a consulta programática", especificamente na categoria "quantidade apresentada". Além disso, foram listados os seguintes itens: procedimentos preventivos coletivos, procedimentos preventivos individuais e procedimentos cirúrgicos. No que diz respeito à coleta de dados referentes às equipes de saúde bucal na ESF, utilizaram-se os dados da Sala de Situação em Saúde do Ministério da Saúde.

Sobre a coleta dos dados referentes à Atenção Especializada, isto é, à provisão de serviços odontológicos, primeiramente realizou-se uma busca dentre os 92 municípios do Estado do Rio de Janeiro, a fim de se registrar quais possuíam CEO, qual era 
o tipo de CEO, bem como a data de habilitação. A partir destes dados, através do SIA-SUS realizou-se uma busca por dados referentes à produção ambulatorial, nos anos de 2004 e 2007, considerando-se procedimentos endodônticos e peridontais.

Quanto ao item fluoretação das águas de abastecimento público, o número de municípios com acesso à água fluoretada, foi obtido através de dados da FUNASA e da Secretaria de Estado da Saúde do Rio de Janeiro.

\section{RESULTADOS}

$\mathrm{Na}$ primeira etapa da análise dos dados, foi descrita a evolução na proporção de municípios que aderiram à PNSB, em relação às três diretrizes programáticas avaliadas neste estudo. Ao comparar os dados de 2010 com os obtidos em 2004, observa-se um incremento expressivo na implantação das ESB e dos CEO, bem como na fluoretação da água (Figura 1).

Em se tratando da cobertura populacional das Equipes de Saúde Bucal, observa-se uma pequena ampliação no período analisado, passando de 23,06\%, em 2004, para 31,25\%, em 2010 (Figura 2).

Para análise do perfil de implantação do PBS nos níveis de atenção básica e especializada, os municípios foram divididos em três grupos, considerando-se o seu porte demográfico: municípios de pequeno porte (com até 30.000 habitantes), de médio porte (de 30.001 habitantes a 100.000 habitantes) e de grande porte (com mais de 100.001 habitantes). Esta divisão por porte foi realizada, pois este é um fator considerado para o estabelecimento de incentivo diferenciado às Equipes de Saúde da Família, conforme a portaria n ${ }^{\circ} 648$ GM/MS (Brasil, 2006).

Ao analisar a evolução da proporção de municípios com ESB na Estratégia Saúde da Família e com CEO entre 2004 e 2010, observou-se que os municípios de pequeno porte foram aqueles que mais implantaram equipes de saúde bucal no primeiro ano. Em contrapartida, os municípios de grande

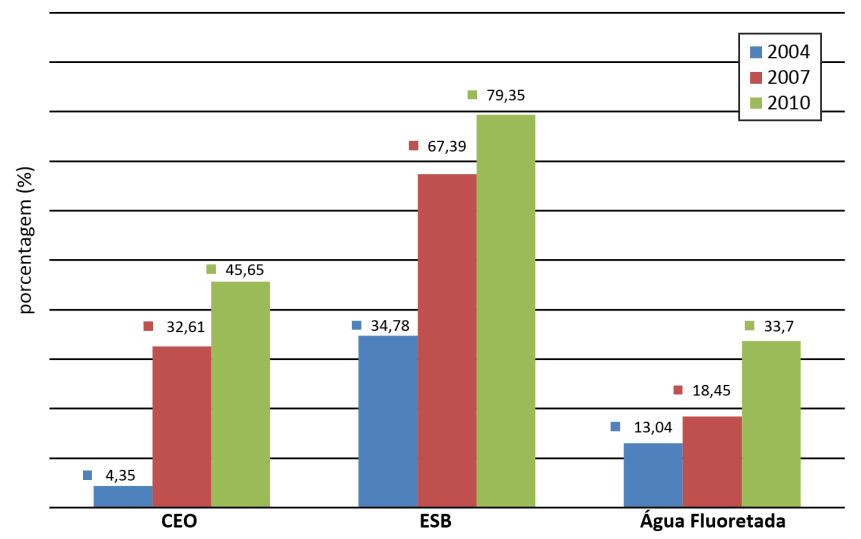

Figura 1. Proporção de municípios cobertos por Centros de Especialidades Odontológicas (CEO), Equipes de Saúde Bucal (ESB) na Estratégia Saúde da Família e fluoretação de águas no Estado do Rio de Janeiro, no período compreendido entre 2004 e 2010 porte não aderiram imediatamente à proposta, porém houve um aumento significativo nos anos subsequentes. Quanto à implantação de CEOs, observa-se um grande incremento nos municípios de médio e grande porte no intervalo entre 2004 e 2007. Por outro lado, diferente do que ocorreu no âmbito da Atenção Básica, a implantação de CEOs nos municípios de pequeno porte mostrou-se lenta (Figura 3).

Em se tratando da evolução da provisão de serviços odontológicos de atenção básica e especializada, segundo a média entre os municípios, observou-se um pequeno aumento

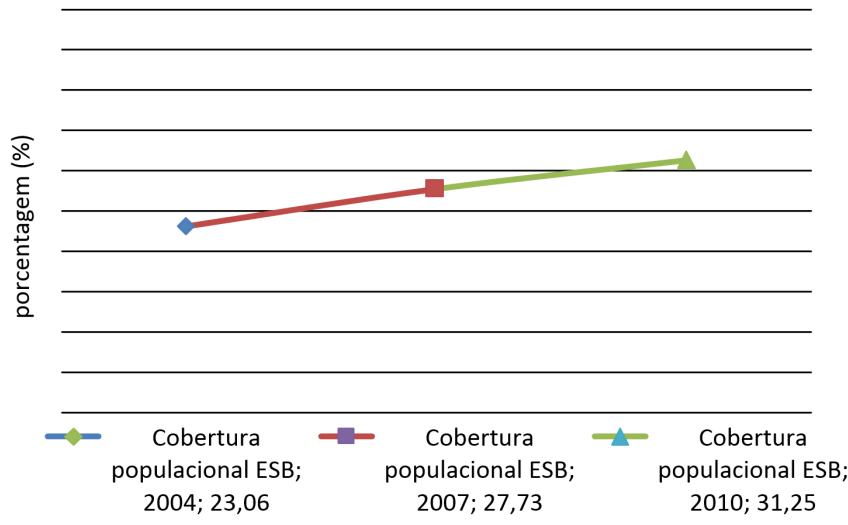

Figura 2. Evolução da cobertura populacional das equipes de saúde bucal na Estratégia Saúde da Família no Estado do Rio de Janeiro entre 2004 e 2010

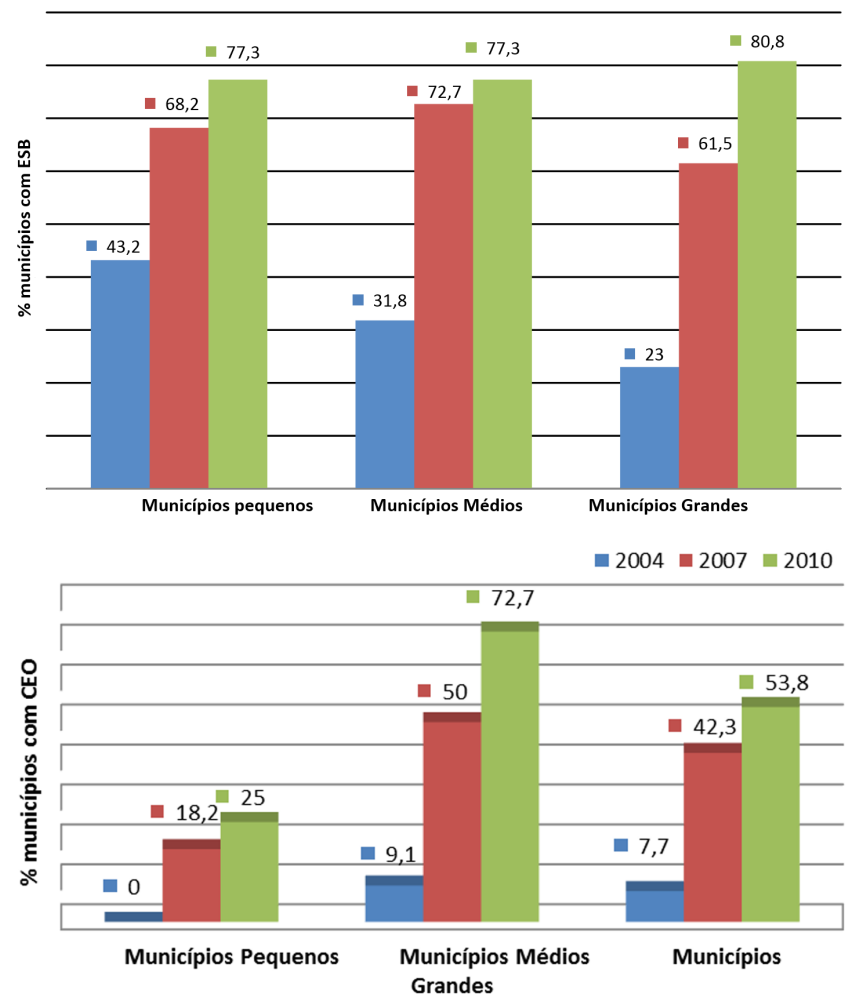

Figura 3. Evolução da implantação das Equipes de Saúde Bucal na Estratégia Saúde da Família e dos Centros de Especialidades Odontológicas (CEO), segundo o porte demográfico dos municípios, entre 2004 e 2010 
nos indicadores relativos às ações básicas em odontologia, às primeiras consultas programáticas, aos procedimentos preventivos individuais, aos procedimentos cirúrgicos e de periodontia. $\mathrm{O}$ indicador relativo às endodontias em dentes permanentes manteve-se constante, sendo que o aumento mais expressivo se deu no indicador referente aos procedimentos preventivos coletivos (Tabela 1).

Ao separar os municípios de acordo com seu porte demográfico, observou-se que a evolução da provisão dos serviços odontológicos se deu de forma diferenciada entre eles. Em relação às primeiras consultas programáticas realizadas por habitante, por exemplo, houve um aumento naqueles de pequeno e grande porte; ao passo que nos de médio porte verificou-se sua redução. Quanto aos procedimentos de atenção básica por habitante, percebeu-se sua ampliação em todos os municípios (Figura 4).

Permanecendo na análise dos indicadores de saúde bucal em relação aos municípios de pequeno, médio e grande porte, observou-se uma ampliação no indicador de procedimentos preventivos coletivos em todos os municípios. Quanto aos procedimentos preventivos individuais e às exodontias, houve sua ampliação nos municípios pequenos e médios e redução nos de grande porte (Figura 5).

\section{DISCUSSÃO}

Com a Política Nacional de Saúde Bucal - Programa Brasil Sorridente, a saúde bucal passou de uma posição inexpressiva para uma de alta centralidade no governo federal ${ }^{4}$. As diretrizes da política propõem a ampliação do acesso a todas as faixas etárias e o atendimento integral em todos os níveis, incentivando estados e municípios a implantarem as ações de saúde bucal na Estratégia Saúde da Família, e a criarem os Centros de Especialidades Odontológicos (CEOs), que funcionariam como unidades de referência de média complexidade para as equipes de saúde bucal, favorecendo a resolutividade. Além disso, outras frentes de atuação do PBS dão enfoque à prevenção e promoção de saúde, incentivando a fluoretação das águas de abastecimento público, dentre outras ações ${ }^{8}$. No presente estudo, observou-se uma ampliação na oferta e provisão de serviços nas três esferas

Tabela 1. Evolução das médias dos procedimentos de Atenção Básica e Especializados em saúde bucal por habitante nos municípios do Estado do Rio de Janeiro entre 2004 e 2007

\begin{tabular}{lrr}
\multicolumn{1}{c}{ Procedimentos } & $\mathbf{2 0 0 4}$ & $\mathbf{2 0 0 7}$ \\
Ações Básicas em Odontologia & 0,21 & 0,22 \\
$1^{\text {a }}$ consulta programática & 1,43 & 1,86 \\
$\begin{array}{l}\text { Procedimentos preventivos coletivos } \\
\text { Procedimentos preventivos }\end{array}$ & 12,81 & 16,15 \\
$\begin{array}{l}\text { individuais } \\
\text { Procedimentos cirúrgicos }\end{array}$ & 0,23 & 0,28 \\
$\begin{array}{l}\text { (exodontias) } \\
\text { Endodontias em dentes permanentes }\end{array}$ & 0,07 & 0,08 \\
Procedimentos de periodontia & 0,12 & 0,01 \\
\hline
\end{tabular}

analisadas (atenção básica, especializada e fluoretação de águas) condizendo com as diretrizes programáticas do PBS.

Em se tratando da viabilização de tratamento e fluoretação das águas, observou-se, neste estudo, um aumento significativo,

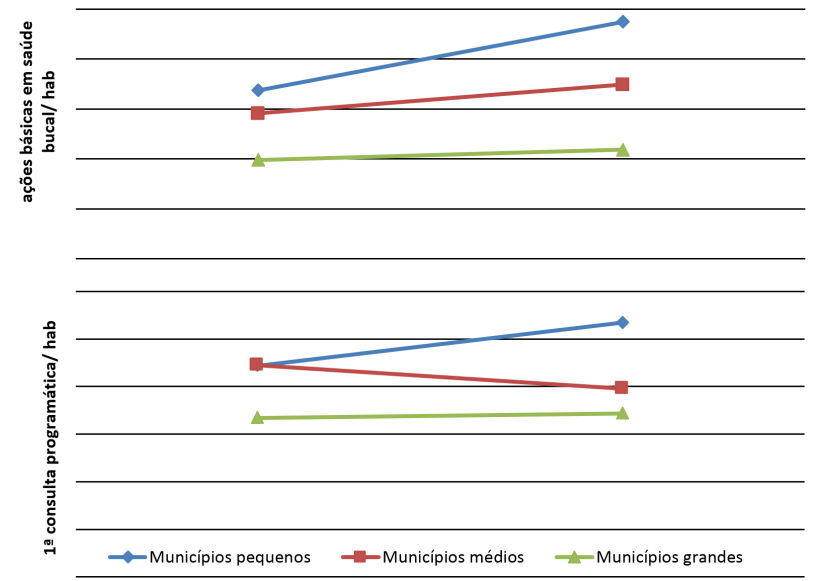

Figura 4. Evolução da provisão de serviços odontológicos no Estado do Rio de Janeiro (ações básicas e primeiras consultas programáticas), segundo o porte demográfico dos municípios, entre 2004 e 2007
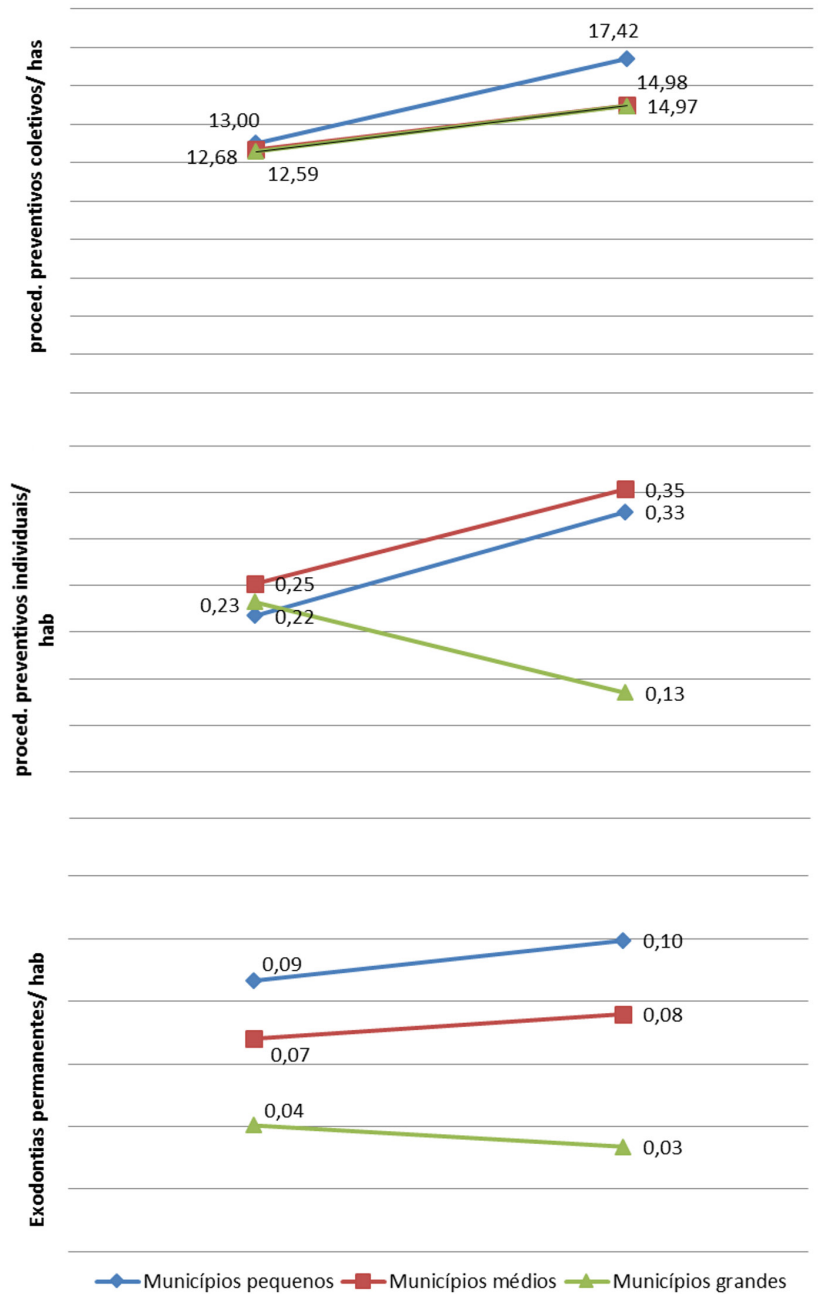

Figura 5. Evolução da provisão de serviços odontológicos no Estado do Rio de Janeiro (procedimentos preventivos e cirúrgicos), segundo o porte demográfico dos municípios, entre 2004 e 2007 
mas ainda não expressivo, em função do número de municípios ainda não incluídos. Embora a fluoretação das águas de abastecimento tenha sido reconhecida como uma efetiva, econômica e abrangente medida de prevenção da cárie dentária em locais de alta prevalência dessa doença ${ }^{9,10}$, sabe-se que, no Brasil, ainda existem municípios que não adicionam flúor à água de abastecimento. Recomendada pela OMS a partir da década de cinquenta, a fluoretação das águas é obrigatória no Brasil, onde exista Estação de Tratamento de Água, conforme estabelecido pela lei federal 6.050, de $1974^{11}$.

Um estudo realizado por Ramos ${ }^{12}$ evidenciou que, em 2013, 30 municípios dos 92 existentes no Rio de Janeiro, ou seja, 37\% eram beneficiados com a fluoretação da água de abastecimento. Comparando este dado com o obtido neste estudo para o ano de 2010 (33,7\%), observa-se que a expansão deste benefício continuou reduzida.

Faz-se necessária, portanto, a ampliação da fluoretação da água nos municípios do Rio de Janeiro, bem como do heterocontrole desta medida. Para Narvai ${ }^{9}$, quando um bem ou serviço pode gerar riscos ou ser um fator de proteção para a saúde, é necessário, além do controle pelo responsável pela sua produção, distribuição e consumo, o heterocontrole pelas instituições do Estado não envolvidas de forma direta neste processo. Assim, preserva-se a sua qualidade, conferindo maior confiabilidade e credibilidade a esta ação, bem como aos seus resultados.

Narvai et al. ${ }^{10}$, ao analisarem a evolução do nível de cárie aos 12 anos, nas 27 capitais estaduais brasileiras, entre 2003 e 2010, observaram um declínio médio nos valores do índice de cárie nas capitais com água fluoretada de 8,6\%, ao passo que naqueles sem acesso à água fluoretada, houve um aumento médio de $12,8 \%$. Tal fato evidencia sua eficácia preventiva. Neste mesmo estudo, foi possível observar que no Estado do Rio de Janeiro, em específico, houve um aumento de 9,45\% deste índice. Tal fato pode estar relacionado à reduzida parcela da população com acesso à água fluoretada. Cumpre destacar que o declínio dos índices de cárie não deve ser atribuído apenas à ação da fluoretação da água, mas também a outras ações propostas pelo Programa Brasil Sorridente.

No que tange à evolução da implantação dos Centros de Especialidades Odontológicas (CEOs), pode-se notar um aumento expressivo destes, porém favorecendo os municípios de médio e grande portes. Resultados similares foram demonstrados por Saliba et al. ${ }^{13}$ para o Brasil, e por Baldani ${ }^{1}$ para o Estado do Paraná. A análise dos municípios por porte demográfico é de grande relevância, na medida em que o funcionamento efetivo dos CEO depende de uma interface apropriada entre a Atenção Primária e a Secundária. Nos municípios de pequeno porte, esta interface depende da pactuação de consórcios com outros municípios para que se assegure o fluxo de referências e contra referências. Caso isto não seja observado, a viabilidade do CEO pode estar comprometida ${ }^{14}$. O pequeno número de CEOs instituídos nos municípios de pequeno porte observado neste estudo pode estar associado a dificuldades no estabelecimento destes consórcios.

Em 2014, o Estado do Rio de Janeiro possuía 42 municípios (45,6\%) com CEO implantado, sendo: 11 municípios de pequeno porte, 14 municípios de médio porte e outros 14 municípios de grande porte.

A incorporação da equipe de saúde bucal na Estratégia de Saúde da Família, em 2000, e o estabelecimento dos Centros de Especialidades Odontológicas, em 2004, representaram novos impulsos para a ampliação da oferta de atendimento odontológico ${ }^{15}$. No Estado do Rio de Janeiro, observou-se um expressivo aumento no número de Equipes de Saúde Bucal (ESBs) implantadas entre 2004 e 2010, chegando a praticamente 80\% dos municípios, reforçando a importância das ESBs para a PNSB no processo de reorganização da atenção básica de saúde do SUS ${ }^{16}$. Mattos et al. ${ }^{17}$ apontam como fatores estimuladores da inserção das equipes de saúde bucal na ESF os incentivos financeiros fornecidos pelo Ministério da Saúde; o fato de os gestores acreditarem na melhoria da saúde bucal da população através deste novo modelo; e a possibilidade de reorganização das ações em saúde bucal fundamentadas na promoção, prevenção e recuperação da saúde.

Apesar do amplo percentual de municípios que implantaram as ESB, observou-se neste estudo uma baixa cobertura populacional no Estado, o que evidenciou que o número de equipes implantadas em cada município ainda é reduzido, necessitando ser ampliado para que beneficiem maior parcela da população.

A ampliação do número de municípios que contam com Equipes de Saúde Bucal e de Centros de Especialidades Odontológicas no Estado justifica o aumento das médias dos procedimentos de Atenção Básica e Especializados em saúde bucal por habitante registrado entre 2004 e 2007. No entanto, para alguns destes indicadores, a ampliação foi mínima. Tal fato pode estar relacionado ao pequeno aumento (cerca de $4 \%$ ) na cobertura das ESB neste período.

Com o aumento no número de municípios com ESB, observou-se ampliação no indicador de $1^{\text {a }}$ consulta programática, em especial nos municípios de pequeno porte, demonstrando maior acesso às consultas odontológicas com a finalidade de diagnóstico e plano de tratamento. Tal fato está em consonância com os achados de Maia e Kornis ${ }^{16}$. Vale destacar que a Política Nacional de Atenção Básica apresenta diferenciação no repasse financeiro para municípios de menor porte populacional e com baixos indicadores de desenvolvimento humano, o que incentiva maior cobertura da Estratégia Saúde da Família, com reflexo direto sobre os indicadores de saúde bucal. Já em relação ao pequeno aumento do indicador $1^{a}$. consulta programática para 
os municípios de maior porte, pode ser também justificado que historicamente são essas as localidades que geralmente apresentam melhor acesso e atenção à saúde bucal. Neste gargalo, encontram-se os municípios com médio porte, que tentam romper o modelo biomédico e, sem um financiamento diferenciado, acabam provocando poucas mudanças na sua forma de organização no acesso e na atenção à saúde bucal.

Em relação aos municípios de médio porte, as ações básicas em saúde bucal aumentaram significativamente, assim como nos municípios de pequeno porte. Por consequência, observou-se aumento nos procedimentos preventivos coletivos e individuais destas cidades. Em contrapartida, nos municípios de grande porte, houve uma diminuição brusca nos procedimentos preventivos individuais e uma discreta diminuição no número de exodontias de dentes permanentes. Observou-se ainda nestes municípios uma pequena ampliação nos indicadores de ações básicas em saúde bucal. Esse pequeno aumento observado em alguns indicadores e a redução de outros podem estar associados à pequena cobertura populacional das ESBs observada no Estado. Destaca-se que a análise do indicador da média de exodontias por habitante é bastante relevante para demonstrar o modelo de saúde adotado pelo município, verificando se está voltado para a promoção de saúde ou se desenvolve somente aspectos mutiladores ${ }^{18}$. Neste sentido, a redução deste indicador se mostra bastante favorável. Ao analisar o indicador exodontia de dentes permanentes, principalmente nos municípios de pequeno e médio porte, constata-se a reprodução histórica do modelo de atenção odontológica com ênfase na assistência curativa e mutiladora, bem como também pelo acúmulo durante vários anos de demanda reprimida por falta de acesso a tratamento odontológico, o que, de certa forma, acaba refletindo na maior necessidade de exodontia em detrimento de procedimentos preventivos e restauradores em saúde bucal.

\section{CONCLUSÃO}

Cerca de onze anos após o lançamento da Política Nacional de Saúde Bucal, podem ser observados grandes avanços na qualidade e abrangência da atenção à saúde bucal, mas também grandes desafios. Os resultados deste estudo permitiram identificar a ampliação na oferta e provisão de serviços odontológicos nos municípios do Estado do Rio de Janeiro a partir de 2004, principalmente da atenção primária, através da inserção das equipes de saúde bucal na ESF; e da atenção secundária, por meio da abertura de Centros de Especialidades Odontológicas. Verificou-se aumento na provisão de serviços por habitante, porém de forma desigual segundo o porte demográfico dos municípios. Além disso, percebeu-se um discreto aumento da população com acesso à agua de abastecimento fluoretada.

No entanto, persistem vários desafios. Dentre eles, destaca-se a necessidade de aumentar a cobertura dessas equipes, a fim de construir uma rede de cuidados mais ampla, que envolva todos os níveis de atenção, a ampliação do número de Centro de Especialidades Odontológica, principalmente nos municípios de pequeno porte e a ampliação da fluoretação da água de abastecimento.

Ressalta-se, ainda, a necessidade de que a Política Nacional de Saúde Bucal seja pensada de forma regionalizada e esteja articulada com as demais políticas públicas, propiciando o desenvolvimento de ações intersetoriais, a superação das desigualdades sociais e o maior acesso às ações e serviços de saúde de forma integral.

\section{REFERÊNCIAS}

1. Baldani MH. A experiência em cidades de médio e pequeno porte do Paraná. In: Moysés ST, Kriger L, Moysés SJ, organizadores. Saúde bucal das famílias: trabalhando com evidências. São Paulo: Artes Médicas; 2008.

2. Aquilante AG, Aciole GG. Construindo um "Brasil Sorridente"? Olhares sobre a implementação da Política Nacional de Saúde Bucal numa região de saúde do interior paulista. Cad Saúde Publica. 2015 Jan;31(1):82-96. http://dx.doi.org/10.1590/0102-311X00193313. PMid:25715294.

3. Pucca Jr GA. A política nacional de saúde bucal como demanda social. Cien Saúde Colet. 2006;11(1):243-6. http://dx.doi.org/10.1590/S141381232006000100033 .

4. Guerra KCM. Os centros de especialidades odontológicas nos municípios do estado do Rio de Janeiro: uma investigação dos fatores identificáveis como facilitadores ou não na implantação de uma política de indução financeira [dissertação]. Rio de Janeiro (RJ): Instituto de Medicina Social, Universidade do Estado do Rio de Janeiro; 2009.

5. Narvai PC. Avanços e Desafios da Política Nacional de Saúde Bucal no Brasil. Tempus. 2011;5(3):21-34.
6. Brasil. Ministério da Saúde. Passo a passo das ações do Brasil Sorridente. Brasília: Ministério da Saúde; 2013.

7. Chaves SCL, Barros SG, Cruz DN, Figueiredo ACL, Moura BLA, Cangussu MCT. Política Nacional de Saúde Bucal: fatores associados à integralidade do cuidado. Rev Saúde Publica. 2010 Dez;44(6):1005-13. http://dx.doi. org/10.1590/S0034-89102010005000041. PMid:20944892.

8. Brasil. Ministério da Saúde. Diretrizes da política nacional de saúde bucal. Brasília: Ministério da Saúde; 2004.

9. Narvai PC. Cárie dentária e flúor: uma relação do século XX. Cien Saúde Colet. 2000;5:381-92.

10. Narvai PC, Frias AC, Fratucci MVB, Antunes JLF, Carnut L, Frazão P. Fluoretação da água em capitais brasileiras no início do século XXI: a efetividade em questão. Saúde Debate. 2014;38(102):562-71.

11. Brasil. Lei no 6.050, de 24 de maio de 1974. Dispõe sobre a fluoretação da água em sistemas de abastecimento quando existir estação de tratamento. Diário oficial da União, Brasília, 17 de maio de 1974. 
12. Ramos JLN. Evolução e panorama atual da cobertura da fluoretação da água de abastecimento público no Brasil, período de 2004 a 2013 [dissertação]. Rio de Janeiro (RJ): Escola Nacional de Saúde Pública Sérgio Arouca; 2013.

13. Saliba NA, Moimaz SAS, Fadel CB, Bino LS. Saúde Bucal no Brasil: uma nova política de enfrentamento para a realidade nacional. Rev Odontol Bras Central. 2010 Abr;18(48):62-6.

14. Machado FCA, Silva JV, Ferreira MAF. Fatores relacionados ao desempenho de Centros de Especialidades Odontológicas. Cien Saúde Colet. 2015;20(4):114963. http://dx.doi.org/10.1590/1413-81232015204.00532014. PMid:25923626.

15. Antunes JLF, Narvai PC. Política de saúde bucal no Brasil e seu impacto social sobre as desigualdades em saúde. Rev Saúde Publica. 2010 Abr;44(2):360-5. http://dx.doi.org/10.1590/S0034-89102010000200018. PMid:20339637.
16. Maia LS, Kornis GEM. A reorganização da atenção à saúde bucal frente aos incentivos federais: a experiência fluminense. Rev APS. 2010;13(1):84-95.

17. Mattos GCM, Ferreira EF, Leite ICG, Greco RM. A inclusão da equipe de saúde bucal na Estratégia Saúde da Família: entraves, avanços e desafios. Cien Saúde Colet. 2014;19(2):373-82. http://dx.doi.org/10.1590/1413 81232014192.21652012. PMid:24863814.

18. Facchini LA, Teixeira ND, Castilho ED. Avaliação da evolução da demanda de saúde bucal através do uso de sistemas de informação em saúde. Rev Enferm Saúde. 2011;1(1):50-9.

Recebido em: Jul. 02, 2015

Aprovado em: Nov. 23, 2015 